\title{
Patient Modeling Using Mind Mapping Representation as a part of Nursing Care Plan*
}

\author{
Hye-Young $\mathrm{Ahn}^{1}$, Eunja Yeon ${ }^{2}$, Eunmi $\mathrm{Ham}^{2}$, and Woojin Paik ${ }^{3, * *}$ \\ ${ }^{1}$ Dept. of Nursing, Eulji University, \\ 143-5 Yongdoo-dong, Jung-gu, Daejeon, 301-832, Korea \\ ahanaya@eulji.ac.kr \\ ${ }^{2}$ Dept. of Nursing Science \\ ${ }^{3}$ Dept of Computer Science, Konkuk University, \\ 322 Danwol-Dong, Chungju-Si, Chungcheongbuk-Do, 380-701, Korea \\ \{eunice, hem2003, wjpaik\}@kku.ac.kr
}

\begin{abstract}
Nursing care plan reports are one of the most important documents in the application of nursing processes. In this paper, we describe how a text discourse analysis and an information extraction system can be used to convert a traditional nursing care plan into a mind mapping representation. Mind mapping is a process to allow the nurses to focus on the patients rather than on a disease process. Mind mapping encourages the nurses to maintain a holistic view of the patient. A mind mapping representation refers to a visual picture of a patient at the center with various nursing care related information visually linked to the patient's form. Our goal is to develop visually browsable models of the patients to aid in the nursing process education and also help the nurses focus on the patients in the actual care settings.
\end{abstract}

\section{Motivation: Information Extraction from Nursing Care Plan}

According to North American Nursing Diagnosis Association (NANDA), a nursing diagnosis is defined as a critical judgment about individual, family, or community responses to actual or potential health problems or life processes. The goal of a nursing diagnosis is to identify health problems of the patient and his/her family. A diagnosis provides a direction for the following nursing care [1]. The nursing care plan as the repositories of knowledge involved in the overall nursing process, which leads up to the nursing diagnosis [2].

A complementary approach to the traditional tabular and narrative based nursing care plan is referred as mind mapping [3]. A mind map is designed to move away from the linear nature of the traditional nursing plan. A mind map is a graphical representation of the connection between concepts and ides that are related to a patient. From the patient representation placed in the middle, associated information, such as nursing diagnosis, expected outcome, suggested interventions, observation summaries, and the evaluation by the nurses, are grouped the linked to the patient. A mind

\footnotetext{
* This paper was supported by Konkuk University in 2005.

** Corresponding author. 
map is for the nurses to develop a whole patient picture that is comprised of a variety of information, which are related to the identified patient problems. A mind map is used to reflect how nurses in practices truly think.

Our text preprocessing program first identifies and extracts the nursing diagnoses, outcomes, and interventions, which are usually in a table. This process is fairly straightforward as each component is placed under clear descriptive heading. However, extracting observed information summaries about the patients and the evaluations by the nurses is difficult. The main focus of the research in this paper is about the extracting the summary and evaluation information in the narrative portion of the nursing care plans. Our text discourse analysis program classifies each clause in the narrative portions of the care plan according to eight categories. When the classification is completed, the semantic relation extraction program identifies the summary and evaluation pairs, which are linked by a causal relation. Then, the causally linked summary and evaluation pairs will become a part of the mind map visual representation. The mind map will be used as a teaching aid for the novice nurses to learn how to evaluate and represent the patient conditions.

\section{Classifying Clauses in Nursing Care Plan Narratives According to a Nursing Process Text Discourse Model}

A text discourse model specifies the necessary classes of knowledge to be identified in order to develop the skeletal conceptual structure for a class of entities. Based on a discourse linguistics observation [4], writers are often influenced by the schema of a particular text type if they produce texts of that type repeatedly. This means that the writers consider both specific content they wish to convey and usual structure for that type of text on the basis of the purpose it is intended to serve.

The existence of such predictable structures in texts is consistent with findings in cognitive psychology which suggest that human cognitive processes are facilitated by the ability to 'chunk' the vast amount of information encountered in daily life into larger units of organized data [5]. Based on schema theories, humans recode individual units of perception into increasingly larger units, which will eventually reach at the level of a schema. It has also been argued that humans possess schema for a wide range of concepts, events, and situations [6]. In discourse linguistics, this schema theory was extended to suggest that schema exist for text-types that participate regularly in the shared communication of a particular community of users.

As the text structure of a particular text type is discovered, the text's discernible and predictable superstructure is also revealed. Superstructure is defined as the textlevel syntactic organization of semantic content. It can be also referred to as the global schematic structure or the recognizable template that is filled with different meaning in each particular example of that text type [7]. Some of the text types for which schemas or models have been developed with varying degree of details are: newspaper articles [7, 11], arguments [8], editorials [9], abstracts [10], and legal texts [12].

To develop a text schema for the narratives in the nursing care plan, we analyzed randomly selected fifteen nursing care plans as a training data set. The care plans were a part of a course assignment for the 'Psychiatric Nursing' course, which was 
offered at the Department of Nursing Science, Konkuk University in Chungju, Korea. The course was for Juniors who were majoring in the nursing science. Each student developed a detailed case study report of one patient while the student was working as a student intern at a psychiatric warden for four weeks. The nursing care plan was one section of the case report, which was submitted at the end of the internship period. The case reports were from the course offered in the Fall 2004 semester. All case reports were mainly written in Korean with English translations for a number of important concepts. We also used another set of fifteen randomly selected nursing care plans as a testing data set.

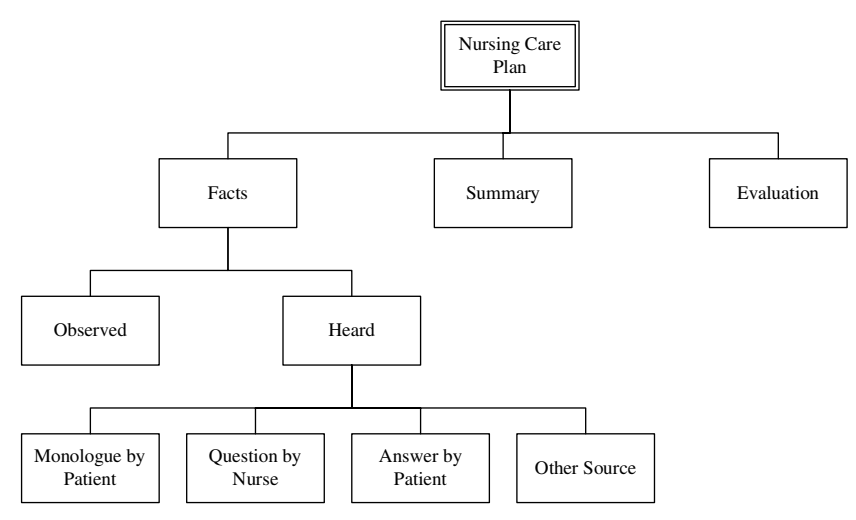

Fig. 1. Nursing Care Plan Text Schema

The nursing care plan text schema is shown in the Figure 1. It is based on the qualitative content analysis of the training data set. At the most general level, there are three major categories. They are 'Facts', 'Summary', and 'Evaluation'. 'Facts' refer to the factual information about the patients. 'Facts' major category is further divided into 'Observed' and 'Heard' categories according to how the information was collected. 'Observed' is for the information directly observed or measured by the nurses. 'Heard' is what the nurses heard about the patient. 'Heard' category is further divided into four sub-categories. Four sub-categories are: 'Monologue by Patient', 'Question by Nurse', 'Answer by Patient', and 'Other Sources'. 'Monologue by Patient' is for the information, which is based on what the patient said about him/herself without external inquiry. 'Question by Nurse' is somewhat problematic sub-category as it does not independently convey information about the patient. Nurses ask questions either to learn a particular aspect about the patient's condition or to encourage the patient to continue talk about him/herself. Thus, most of the clauses classified under this category do not convey substantive information. 'Answer by Patient' subcategory is for the information provided by the patient in response to a question by the attending nurses. Finally, 'Other Sources' sub-category refers to the patient information provided by the family members, friends, other nurses, or the physicians.

We decided to code each clause with the discourse categories at the most specific level. The following shows two paragraphs in two different nursing care plans. These plans were used as the training data set. Although, all qualitative content analysis and 
computational processing is directly applied to the original Korean text, the example paragraphs are manually translated into English to help non-Korean to understand the meaning of the examples. '<category name>' and '</category name>' are used to show the beginning and end of a clause, which is coded as a particular discourse category.

Example 1. The example is from a paragraph explaining the insight of a patient HS in the mental state assessment section.

<monologueByPatient> HS said 'I hope my nervousness goes away soon'. $</$ monologueByPatient $><$ evaluation $>$ Based on the statement, HS seems to understand about his condition well. </evaluation $><$ otherSource $>$ As HS voluntarily admitted to the hospital last time, </otherSource $><$ evaluation $>$ it is believed that HS is positively thinking about the treatments that HS is receiving. $<$ evaluation $>$

Example 2. The example is from a paragraph describing the life when the patient JK was in teens in the developmental history part.

<questionByNurse> Nurse asked 'Have you ever had a girl friend?' $</$ questionByNurse> < answerByPatient> JK said 'No, I never had a girl friend.' $<$ lanswerByPatient> <questionByNurse> Nurse asked 'Do you have many friends?' </questionByNurse > <answerByPatient> JK said 'No, I do not have many friends.' </answerByPatient $><$ summary $>$ As the patient stated that he never had a relationship with a female and also did not have many male friends, $</$ summary $><$ evaluation $>$ it is likely that $J K$ have been having a difficult interpersonal relationship since he was a teenager. </evaluation $>$

\subsection{Extracting Text Classification Features}

While coding the training data, we developed both defining features and properties for each category. The defining features convey the role and purpose of that category within the nursing care plan text schema while the properties provide suggestive clues for the recognition of that category. The manual coding suggested to us that we were relying on five types of linguistic information during our coding. The data, which would provide these evidence sources, were then analyzed statistically and translated into computationally recognizable text characteristics. The five sources of evidences are described in the following.

Lexical Evidences: This source of evidence is a set of one, two, three word phrases for each component. The set of lexical evidences for each component was chosen based on observed frequencies and distributions. Only the words or phrases with sufficient occurrences and statistically skewed observed frequency of occurrences in a particular component were used.

After all coded training data are processed by the lexical evidence extraction module, the frequency distribution of each piece of lexical evidence is further processed to generate the probability information. For example, '있는 듯 하다 (English translation: it seems to be)' occurred three times in the clause coded as 'Facts', six times in the 'Summary' clauses, and 15 times in 'Evaluation' in the training data set. This 
tells us that the clause should be coded as 'Facts' three out of 24 times if the sentence includes '있는 듯 하다' as an example of three word lexical evidences. In the same manner, '있는 듯 하다' is indicated six out of 24 times as 'Summary', and 15 out of 24 times as 'Evaluation'. After all one, two, and three-word lexical evidence based on probability calculation is completed, a series of normalization processes is applied to weed out unreliable lexical evidences and also to remove other influences such as likelihood of component occurring. As each clause is processed, the lexical evidences for the words and phrases in the clause are combined using the Dempster-Shafer Theory of Evidence [13]. At the end of all processing, each clause is assigned with four numbers ranging from zero to one. One number represents the clause's probability of having been correctly coded as 'Facts'. Other numbers are the probability figures for 'Summary', and 'Evaluation'.

Syntactic Evidences: We utilize two types of syntactic evidences: 1) typical sentence length as measured in the average number of words per clause for each category and 2) individual part-of-speech distribution based on the output of the part-of-speech tagging. This evidence helps to recognize those categories, which tend to have a disproportionate number of their words be of a particular part of speech. For example, 'Observed' category clauses tend to have comparatively small number of adjectives.

Tense Evidences: Some components tend to contain verbs of a particular tense more than verbs of other tenses. For example, 'Fact' clauses are almost always in the past or present perfect tense. The tense evidence is a byproduct of part-of-speech tagging.

Document Structure Evidences: There are two types of document structure evidences. Firstly, nursing care plans have section headings, which are closely aligned with three major categories. For example, 'Health related Information' section rarely had clauses, which are categorizes as 'Summary' or 'Evaluation'. Thus, we utilized the heading information as another evidence source. Secondly, we included the relative position of each clause with respect to the source paragraph as another evidence source.

Order of Component Evidences: This source of evidence replies on the tendency of categories to occur in a particular, relative order. We calculated the frequency with which each category followed every other category and the frequency with which each category preceded every other category. The results are stored in two seven-byseven matrices.

\subsection{Text Classification}

To assign a basic category label to each clause, each clause in the training data set is categorized according to the predetermined nursing care plan text schema. The first text classification task involves manually coding all clauses in a set of training documents in preparation for feeding the automatic system. Each clause is classified as "in" or "out" of the individual categories as outlined by the category definitions. The next step is to take these manually classified clauses and process them through the 
trainable text classification system. During this process, it builds a vector of lexical evidences, syntactic evidences, tense evidences, and document structure evidences. Multi-level Natural Language Processing outputs are the basis for these textual data feature representations.

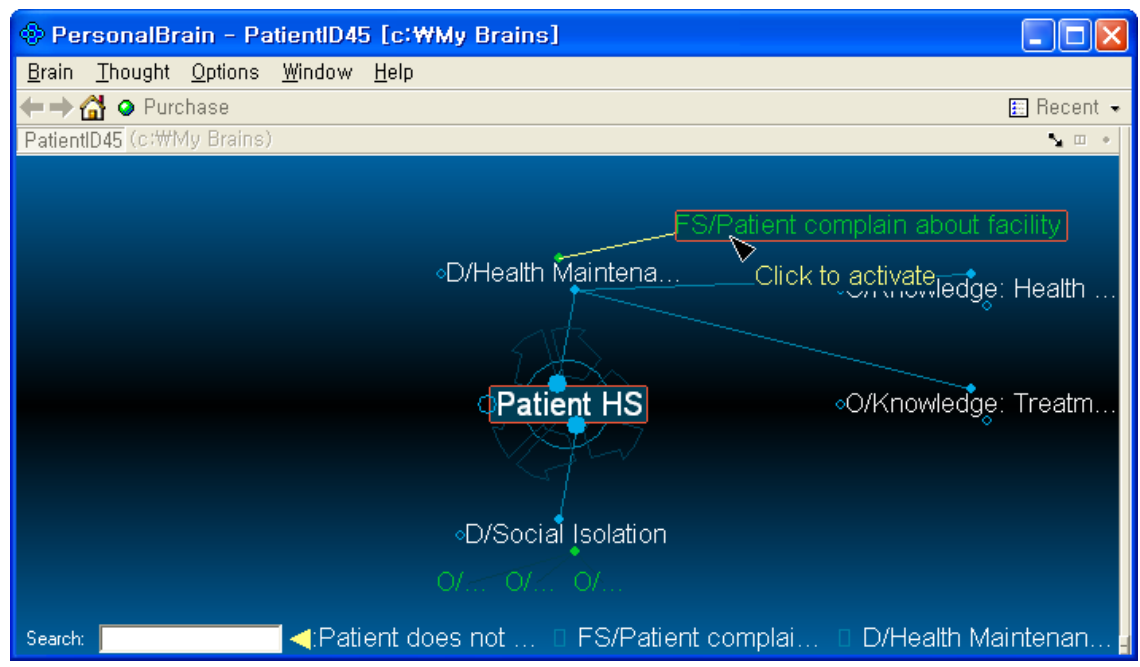

Fig. 2. Mind Map for Patient HS

\section{Making a Mind Map Visual Representation}

Our goal is to make a mind map of a patient along with all related factual and evaluative information about the patient. The Figure 2 shows a mind map representation about a patient, who is referred as HS.

For this research, we used PersonalBrain, a commercial off the shelf software from The Brain Technologies Corp (http://www.thebrain.com/). PersonalBrain is an information visualization system, which enables the users to link information into a network of logical associations. PersonalBrain uses 'Thoughts' to refer 'concepts' and 'Links' to refer 'relations'. The Figure 2 shows 'Health Maintenance, Altered' and 'Social Isolation' as the nursing diagnoses for the patient HS. Each nursing diagnosis is preceded by ' $\mathrm{D}$ '. Nursing Outcome is preceded by ' $\mathrm{O}$ ' and Intervention is preceded by 'I'. PersonalBrain truncates the labels of each node in the graph if the label is too long. The full label is revealed when the user moves the cursor on top of the node. The user can make any node a central node by double clicking on it. The mind map represented in PersonalBrain is dynamically repositioned to minimize the overloading of the visual representation in small screen space. At the top of the mind map, there is a factual information summary observed by the nurse. "Patient complain about facility' is preceded by 'FS', which stands for 'factual summary'. ' $E$ ' is for the evaluative statement by the nurses. 


\section{Implementation and Evaluation}

The computational modeling of instantiating a discourse-level model of the nursing care plan texts and converting the traditional nursing care plan to the mind map visual representation is an ongoing effort. We developed a prototype system by manually analyzing fifteen sample care plans and tested our system using fifteen unseen care plans. The first run and evaluation of the correctly categorizing four basic categories resulted in $80 \%$ of the sentences being correctly identified.

There is no directly comparable nursing care plan text classification system. However, a news text classification system, which assigned sentences into one out of fourteen categories, performed at $72 \%$ correct rate in the fully automatic mode and $80 \%$ correct rate with various manual heuristic adjustments [11]. It should be noted that our text classifier did not utilize the second iteration of incorporating the sentences, with certainty membership value close to zero, as a part of new training data set. We believe the addition of this process will improve the correctness of our system.

\section{Summary}

Although we are clearly in the early stages of developing a patient modeling system based on the mind map representation scheme through the use of nursing care plan discourse modeling and information extraction system, we find these results quite promising and eager to share our premature but empirical results and experiences in creating an operational system with other researchers.

We expect the resulting mind map to aid both nursing students and practitioners finding the nursing process examples of making proper nursing diagnosis. They can review what others have done to learn from the sound decisions and the mistakes.

There are many tasks that we have yet to finish. The major missing task is the usability evaluation of the resulting mind map. We also need to increase the size of test data set to improve the reliability of the evaluation results.

We have applied the nursing care plan discourse model to the actual care plans by coding a small set of sample texts. This effort in conjunction other automatic discourse modeling works, we argue that it is possible to extract a particular section of the texts by utilizing a text type specific discourse model.

\section{References}

1. Sparks. S.M. and Taylor, C.M., Nursing Diagnosis Reference Manual 5th Edition, Springhouse, Springhouse, Pennsylvania, 2000.

2. Doenges, M., and Moorehead, M.F., Application of Nursing Process and Nursing Diagnosis: An Interactive Text for Diagnostic Reasoning $4^{\text {th }}$ Edition, F.A. Davis Co., Philadelphia, Pennsylvania, 2003.

3. Mueller, A., Johnston, M. \& Bligh, D.: Joining mind mapping and care planning to enhance student critical thinking and achieve holistic nursing care. Nursing Diagnosis 13(1):24-27 (2002).

4. Jones, L.B.: Pragmatic aspects of English text structure. Summer Institute of Linguistics, Arlington, TX (1983). 
5. Rumelhart, D.: Understanding and summarizing brief stories. In D. LaBerge and S.J. Samuels (Editors) Basic processes in reading: Perception and comprehension. Hillsdale, NJ: Lawrence Earlbaum Associates: 265-303. (1977)

6. Rumelhart, D.: Schemata: the building blocks of cognition. In R. Spiro, B. Bruce, \& W. Brewer (Editors) Theoretical issues in reading comprehension: Perspectives from cognitive psychology, linguistics, artificial intelligence and education. Hillsdale, NJ: Lawrence Earlbaum Associates: 33-8. (1980)

7. van Dijk, T.A.: News analysis: Case studies of international and national news in the press. Hillsdale, NJ: Lawrence Earlbaum Associates. (1988)

8. Cohen, R.: Analyzing the structure of argumentative discourse. Computational Linguistics, 13. 11-24. (1987)

9. Alvarado, S.J.: Understanding editorial text: A computer model of argument comprehension. Boston, MA: Kluwer Academic Publishers. (1990)

10. Liddy, E.D.: The discourse-level structure of empirical abstracts: An exploratory study. Information Processing and Management 27.1, Tarry Town, NY, Pergamon Press: 55-81. (1991)

11. Liddy, E.D., McVearry, K.A., Paik, W., Yu, E., and McKenna, M.: Development, Implementation and Testing of a Discourse Model for Newspaper Texts. Proceedings of a Human Language Technology Workshop. Plainsboro, NJ, Morgan Kaufmann Publishers: 159-164. (1993)

12. Paik, W. and Lee, J.: Extracting Legal Propositions from Appellate Decisions with Text Discourse Analysis Methods. Lecture Notes in Computer Science (LNCS) Vol. 3292, Springer-Verlag: 621-633. (2004)

13. Pearl, J.: Probabilistic reasoning in intelligent systems: networks of plausible inference, Morgan Kaufmann Publishers, San Francisco, CA. (1988) 